PROCEEDINGS OF THE

AMERICAN MATHEMATICAL SOCIETY

Volume 126, Number 6, June 1998, Pages 1669-1671

S 0002-9939(98)04236-1

\title{
A VERSION OF STRASSEN'S THEOREM FOR VECTOR-VALUED MEASURES
}

\author{
A. HIRSHBERG AND R. M. SHORTT \\ (Communicated by Clifford J. Earle, Jr.)
}

\begin{abstract}
A formulation of Strassen's Theorem is given for measures taking values in a Banach lattice. The main result (Theorem 2) corrects earlier work of the second author.
\end{abstract}

In joint work of M. März and the second author [5, Theorem 3.7], a version of the theorem known in probability theory as "Strassen's Theorem" (see [8], [2, 11.6]) was generalized to the context of measures assuming values in a reflexive Banach lattice. In [7, Theorem 3.2], the second author announced an extension of the result to all order-complete Banach lattices. However, the proof given in [7, p.816] contains an error, leaving the validity of the result an open question.

In Theorem 2 below, a result of this general type is proved for measures taking values in Banach lattices of a certain type: the so-called KB-spaces. The KBspaces occupy a position between the reflexive and the complete Banach lattices (reflexive $\Rightarrow \mathrm{KB} \Rightarrow$ complete), so that Theorem 2 is a generalization of $[5$, Theorem 3.7], but is not as strong as what was asserted in [7]. Our technique makes use of a Strassen-type result (given below as Theorem 1 and proved in [3]) for finitely additive measures with values in a complete Banach lattice. As a general reference for basic facts about vector measures, we recommend the text of Diestel and Uhl [1]. For Banach lattices, see [4], [6].

If $\mathcal{A}$ and $\mathcal{B}$ are fields on sets $X$ and $Y$, respectively, then $\mathcal{A} \times \mathcal{B}$ is the field on $X \times Y$ generated by all rectangles $E \times F$ for $E \in \mathcal{A}$ and $F \in \mathcal{B}$. The following result is Theorem 2.1 in [3]. It replaces the imprecisely stated and incompletely proved Theorem 2.2 of [7].

Theorem 1. Let $\mathcal{A}$ and $\mathcal{B}$ be countable fields on sets $X$ and $Y$ respectively and let $\mu: \mathcal{A} \rightarrow G^{+}$and $\nu: \mathcal{B} \rightarrow G^{+}$be finitely additive measures taking values in the positive cone of a divisible, $\sigma$-complete, partially ordered group $G$. We assume that $\mu(X)=\nu(Y)=\alpha$ for some $\alpha \in G^{+}$. Let $S$ be an arbitrary subset of $X \times Y$ and let $\mathcal{C}$ be the field on $X \times Y$ generated by $S$ and the sets in $\mathcal{A} \times \mathcal{B}$. For an element $v \in G$ with $0 \leq v \leq \alpha$, we consider the following conditions:

i) There is a finitely additive measure $\rho: \mathcal{C} \rightarrow G^{+}$such that $\rho(E \times Y)=\mu(E)$ and $\rho(X \times F)=\nu(F)$ for all $E \in \mathcal{A}$ and $F \in \mathcal{B}$ (i.e. $\rho$ has marginals $\mu$ and $\nu$ ) and such that $\rho(S)=v$.

ii) Whenever $E \times F \subseteq S$ for $E \in \mathcal{A}$ and $F \in \mathcal{B}$, then $\mu(E)+\nu(F) \leq \alpha+v$.

Received by the editors July 24, 1996 and, in revised form, October 28, 1996.

1991 Mathematics Subject Classification. Primary 28B05; Secondary 30C62, 46B42.

(C)1998 American Mathematical Society 
iii) Whenever $E \times F \subseteq S^{c}$ for $E \in \mathcal{A}$ and $F \in \mathcal{B}$, then $\mu(E)+\nu(F) \leq 2 \alpha-v$. Then $i)$ is equivalent to the conjunction of ii) and iii).

Let $\mathcal{A}$ be a field of subsets of a set $X$ and suppose that $\mu: \mathcal{A} \rightarrow B$ is a finitely additive measure taking values in a Banach space $B$. Then $\mu$ is called strongly additive if the series $\sum \mu\left(E_{n}\right)$ converges in $B$ whenever $\left(E_{n}\right)_{n}$ is a sequence of pairwise disjoint sets drawn from $\mathcal{A}$. For further information on strong additivity, see [1]. Every non-negative real-valued measure is strongly additive, but non-negative measures taking values in a partially ordered Banach space may fail to be strongly additive, even if they are countably additive.

In [7], a Strassen Theorem for countably additive vector measures taking values in the positive cone of a complete Banach lattice was announced [7, Theorem 3.2]. Unfortunately, there was a gap in the proof of that result, viz. the appeal to the extension theorem of Kluvanek [1, Theorem 2, p.27] made at the top of p. 816 is improper, there being no guarantee that the measure $\rho_{0}$ is strongly additive. The authors have not been able to repair this breach; neither has anyone discovered a counter-example. Thus, no answer has yet been found to the open

Question. Is the formulation of Strassen's Theorem given in [7, Theorem 3.2] valid?

In view of all this, we now seek further conditions on a Banach lattice $(B, \leq)$ sufficient to yield a reasonable Strassen Theorem for countably additive $B$-valued measures. Our approach here is to restrict attention to the so-called KB-spaces: a Banach lattice $(B, \leq)$ is a $K B$-space if each norm bounded increasing sequence $\left(x_{n}\right)_{n}$ in $B$ is convergent. For information on these spaces, see the books of Vulikh [9, p.188ff.], Schaefer [6, p. 92 et seq.] and Zaanen [10, Chapter 15]. In these sources are to be found the following results.

Fact. The KB-spaces are precisely the Banach lattices that contain no subspace isomorphic (as a Banach space) to $c_{0}$, the space of all real sequences converging to zero.

Fact. Every $K B$ space $(B, \leq)$ is complete. In fact, for any $A \subseteq B$ having a supremum $x$, there is a countable $A_{0} \subseteq A$ such that $x=\sup \left(A_{0}\right)$. (The latter property is called super Dedekind completeness.)

Fact. Every reflexive Banach lattice is a KB-space; so too is every Banach lattice $(B, \leq)$ of type $(L)$, i.e. such that $\|x+y\|=\|x\|+\|y\|$ for all $x, y \geq 0$.

Thus, spaces of the form $L^{1}(X, \mathcal{A}, m)$ (e.g. $\left.\ell^{1}\right)$ are KB-spaces, but are not reflexive unless they are finite-dimensional.

Lemma. Let $\mathcal{A}$ be a field of subsets of a set $X$. Then every finitely additive measure $\mu: \mathcal{A} \rightarrow B^{+}$taking values in the positive cone of a $K B$-space $(B, \leq)$ is strongly additive.

Proof. If $\left(E_{n}\right)_{n}$ is a sequence of pairwise disjoint sets in $\mathcal{A}$, then the sequence of partial sums $s_{N}=\sum_{n=1}^{N} \mu\left(E_{n}\right)$ is increasing and is bounded above by $\mu(X)$. Thus the infinite series $\sum \mu\left(E_{n}\right)$ converges.

It is this result that enables us to patch, though not fully repair, the error in [7]. If $\mathcal{A}$ and $\mathcal{B}$ are $\sigma$-fields of subsets of sets $X$ and $Y$, respectively, then $\mathcal{A} \otimes \mathcal{B}$ is the $\sigma$-field on $X \times Y$ generated by $\mathcal{A} \times B$. 
Theorem 2. Let $\mathcal{A}$ and $\mathcal{B}$ be $\sigma$-fields of subsets of sets $X$ and $Y$, respectively, and let $\mu: \mathcal{A} \rightarrow B^{+}$and $\nu: \mathcal{B} \rightarrow B^{+}$be countably additive vector measures taking values in the positive cone of a $K B$-space $(B, \leq)$ and such that $\mu(X)=\nu(Y)=\alpha$. Suppose that $\mu$ is a perfect measure (see [7]) and that $S \in \mathcal{A} \otimes \mathcal{B}$ is a countable intersection of sets in $\mathcal{A} \times B$. For any $v \in B^{+}$, the following are equivalent:

i) There is a $\sigma$-additive measure $\rho: \mathcal{A} \otimes \mathcal{B} \rightarrow B^{+}$with marginals $\mu$ and $\nu$ such that $\rho(S) \geq v$.

ii) For all $E \in \mathcal{A}$ and $F \in \mathcal{B}$, we have $\mu(E)+\nu(F) \leq 2 \alpha-v$ whenever $E \times F \subseteq S^{c}$.

Proof. i) $\Longrightarrow$ ii): This is just as in Theorem 1 .

ii) $\Longrightarrow$ i): Define $I=\inf \left\{2 \alpha-\mu(E)-\nu(F): E \times F \subset S^{c}\right\}$ and

$$
\Sigma=\sup \{\mu(E)+\nu(F)-\alpha: E \times F \subset S\} .
$$

We now show that $\Sigma \leq I$ : suppose that $E_{1} \times F_{1} \subset S$ and $E_{2} \times F_{2} \subset S^{c}$. Note that either $E_{1} \cap E_{2}=\emptyset$ or $F_{1} \cap F_{2}=\emptyset$; it follows that $\mu\left(E_{1}\right)+\nu\left(F_{1}\right)+\mu\left(E_{2}\right)+\nu\left(F_{2}\right) \leq 3 \alpha$, and hence that $\mu\left(E_{1}\right)+\nu\left(F_{1}\right)-\alpha \leq 2 \alpha-\mu\left(E_{2}\right)-\nu\left(F_{2}\right)$, as desired. Let $v_{0}=v \vee \Sigma$. It can be seen that $\Sigma \leq v_{0} \leq I$, so that conditions ii) and iii) of Theorem 1 hold with $v_{0}$ in place of $v$. Let $\mathcal{C}$ be the field generated by $\mathcal{A} \times B$ and the set $S$. By Theorem 1 , there is a finitely additive measure $\rho_{0}: \mathcal{C} \rightarrow B^{+}$with marginals $\mu$ and $\nu$ and such that $\rho_{0}(S)=v_{0}$. Now the restriction of $\rho_{0}$ to $\mathcal{A} \times B$ has countably additive marginals, one of which is perfect, so that [7, Theorem 3.1] implies that $\rho_{0}$ is $\sigma$-additive on $\mathcal{A} \times \mathcal{B}$. Using Lemma 3.3 and Kluvanek's Theorem [1, p. 27], we find a $\sigma$-additive measure $\rho: \mathcal{A} \otimes \mathcal{B} \rightarrow B^{+}$such that $\rho=\rho_{0}$ on $\mathcal{A} \times B$. Choose sets $C_{n} \in \mathcal{A} \times B$ such that $C_{n} \downarrow S$ as $n \rightarrow \infty$. We reckon

$$
\rho(S)=\lim \rho\left(C_{n}\right)=\lim \rho_{0}\left(C_{n}\right) \geq \rho_{0}(S)=v_{0} \geq v,
$$

establishing the theorem.

\section{REFERENCES}

1. J. Diestel and J. J. Uhl, Jr., Vector Measures, Mathematical Surveys, No. 15, Amer. Math. Soc., Providence, RI, 1977. MR 56:12216

2. R. M. Dudley, Real Analysis and Probability, Wadsworth \& Brooks/Cole, Pacific Grove, 1989. MR 91g:60001

3. A. Hirshberg and R. M. Shortt, Strassen's Theorem for group-valued charges, pre-print.

4. J. L. Kelley, J. Namioka, et al., Linear Topological Spaces, Van Nostrand, Princeton, Reprinted by Springer-Verlag, New York, 1976. MR 52:14890

5. M. März and R. M. Shortt, Weak convergence of vector measures, Publicationes Math. 45 (1994), 71-92. MR 96g:28015

6. H. H. Schaefer, Banach Lattices and Positive Operators, Springer-Verlag, Berlin, 1974. MR 54:11023

7. R. M. Shortt, Strassen's Theorem for vector measures, Proc. Amer. Math. Soc. 122 (1994), 811-820; Correction, Proc. Amer. Math. Soc., this number. MR 95a:28005

8. V. Strassen, The existence of probability measures with given marginals, Ann. Math. Stat. 36 (1965), 423-439. MR 31:1693

9. B. C. Vulikh, Introduction to the Theory of Partially Ordered Spaces, Wolters-Noordhoff, Groningen, 1967. MR 37:121

10. A. C. Zaanen, Riesz Spaces II, North-Holland, Amsterdam, 1983. MR 86b:46001

Department of Mathematics, Wesleyan University, Middletown, Connecticut 064590128 